\title{
La dirección escolar: entre estrategias de inclusión y \\ mecanismos de control
}

\author{
Yelicich,Carolina \\ CONICET, UNC/UMA, Córdoba,Argentina \\ carolinayelicich@hotmail.com
}

\section{Resumen}

La dirección escolar se ha convertido en objeto de estudio con múltiples abordajes teóricos en las últimas décadas. Estos estudios se han producido a la luz de procesos de reestructuración del papel del Estado, gracias a la implementación de políticas de corte neoliberal con fuerte sesgo mercantilista, que han transformando sustancialmente los modos de trabajo y con ellos, las exigencias a los sistemas educativos. Los centros escolares se ven interpelados diariamente por un mercado que exige e impone sus reglas.

El mercado reconoce la fuerza de los sistemas educativos para generar sujetos adaptados a las nuevas reglas de producción y el sistema productivo intenta así trasladar sus formas de trabajo hacia los sistemas educativos. En este contexto, el trabajo con planes, programas y proyectos se extiende con fuerza como una nueva manera de abordar la tarea de las escuelas. Es posible identificar que los planes, programas y proyectos se transforman por un lado, en modos alternativos de hacer frente a las carencias que sufren tanto alumnos y docentes, como las escuelas en su conjunto, atender a la diversidad e intentar palear la desigualdad y la exclusión en múltiples aspectos. No obstante, estos programas focalizados y asociados a financiamiento, se manifiestan también como nuevos mecanismos de control que sustituyen los formatos tradicionales de rendición de cuentas, para introducir nuevas instancias de regulación del accionar de las instituciones educativas, en el marco de un doble juego en donde los Estados se desligan de la responsabilidad por el funcionamiento de los sistemas educativos - los centros educativos, los directivos y los docentes son los responsables - pero se apropian cada vez con mayor fuerza de la evaluación y el requerimiento de resultados.

Presentados como estrategias para sostener la obligatoriedad escolar, lograr equidad y apuntalar la inclusión educativa, los planes, programas y proyectos enmascaran procesos de evaluación y control centrado en el rendimiento. El objetivo de esta comunicación es examinar estos nuevos mecanismos de trabajo en las escuelas y el papel clave de los directores en la gestión de los mismos, analizando comparativamente un instituto secundario de la ciudad de Málaga, España y una escuela secundaria de la ciudad de Córdoba, Argentina.

\section{Abstract}

School management has become an object of study with multiple theoretical approaches in recent decades. These studies have been produced in light of processes of restructuring the role of the State, thanks to the implementation of neo-liberal policies with a strong mercantilist bias, which have substantially transformed the work modes and, with them, the demands on the education systems. The schools are questioned daily by a market that demands and imposes its rules.

The market recognizes the strength of educational systems to generate subjects adapted to the new rules of production and the productive system thus tries to transfer their forms of work to educational systems. In this context, the work with plans, programs and projects is spreading strongly as a new way of approaching the task of the schools. It is possible to identify that plans, programs and projects are transformed on the one hand, in alternative ways of dealing with the shortcomings suffered by students and teachers, as well as schools as a whole, addressing diversity and trying to shovel inequality and exclusion in multiple aspects. However, these targeted programs associated with financing also manifest themselves as newcontrol mechanisms that replace the traditional forms of accountability, to introduce new instances of regulation of the actions of educational institutions, within the framework of a double game in where the States dissociate themselves from the responsibility for the functioning of the educational systems - the educational centers, the managers and the teachers are responsible - but they take over the evaluation and the requirement of results with greaterforce.

Presented as strategies to sustain the compulsory school, achieve equity and underpin educational inclusion, plans, programs and projects mask processes of evaluation and control focused on performance. The objective of this communication is to examine these new mechanisms of work in schools and the key role of the directors in their management, comparatively analyzing a secondary school in the city of Malaga, Spain and a secondary school in the city of Córdoba , Argentina.

Palabras clave: Dirección escolar, Director escolar, Planes, Regulación.

Keywords: School management, School director, Plans, Regulation. 


\section{INTRODUCCIÓN}

La dirección escolar se ha convertido en objeto de estudio con múltiples abordajes teóricos en las últimas décadas. Estos estudios se han producido a la luz de procesos de reestructuración del papel del Estado, gracias a la implementación de políticas de corte neoliberal con fuerte sesgo mercantilista, que han transformando sustancialmente los modos de trabajo y con ellos, las exigencias a los sistemas educativos. Los centros escolares se ven interpelados diariamente por un mercado que exige e impone sus reglas.

El mercado reconoce la fuerza de los sistemas educativos para generar sujetos adaptados a las nuevas reglas de producción y el sistema productivo intenta así trasladar sus formas de trabajo hacia los sistemas educativos. En este contexto, el trabajo con planes, programas y proyectos se extiende con fuerza como una nueva manera de abordar la tarea de las escuelas. Es posible identificar que los planes, programas y proyectos se transforman por un lado, en modos alternativos de hacer frente a las carencias que sufren tanto alumnos y docentes, como las escuelas en su conjunto, atender a la diversidad e intentar palear la desigualdad y la exclusión en múltiples aspectos. No obstante, estos programas focalizados y asociados a financiamiento, se manifiestan también como nuevos mecanismos de control que sustituyen los formatos tradicionales de rendición de cuentas, para introducir nuevas instancias de regulación del accionar de las instituciones educativas, en el marco de un doble juego en donde los Estados se desligan de la responsabilidad por el funcionamiento de los sistemas educativos - los centros educativos, los directivos y los docentes son los responsables - pero se apropian cada vez con mayor fuerza de la evaluación y el requerimiento de resultados.

Mecanismos presentados como estrategias para sostener la obligatoriedad escolar, lograr equidad y apuntalar la inclusión educativa, enmascaran procesos de evaluación, control y direccionamiento de las tareas de los centros escolares para el cumplimento de objetivos cuantificables y observaciones. Eficacia y eficiencia, conjuntamente con el logro de índices de calidad, allanan el camino para la extensión de la privatización y mercantilización de los sistemas educativos. La mercado adopta así como nuevo aliado al Estado.

\section{NUEVAS FORMAS DE REGULACIÓN EN LA ESCUELA}

En el ámbito educativo, la reforma del Estado que se está produciendo desde hace décadas, ha dado lugar a nuevas y múltiples fuentes de regulación de la actividad escolar. En este sentido, la regulación implica tanto el conjunto de acciones y reglamentaciones que dispone una instancia para orientar el accionar de los individuos como así también la reorganización y las estrategias que se despliegan a partir de lo reglado (Miranda y Lamfri, 2008). Barroso (2005), en una interesante revisión sobre la pluralidad de significados del concepto, plantea que la regulación puede ser entendida como «la intervención de las autoridades gubernamentales en la prestación de un servicio público» (Barroso, 2005, p. 733). Complejiza la mirada luego, al afirmar que la regulación

comprende no sólo la definición de reglas (normas, mandatos, restricciones, etc.) que orientan el funcionamiento del sistema, sino también su (re)ajuste provocado por la diversidad de estrategias y acciones de los diversos actores, en función de esas mismas reglas. De acuerdo con este abordaje, en un sistema social complejo (como es el sistema educativo) existe una pluralidad de fuentes, de finalidades y modalidades de regulación, en función de la diversidad de los actores involucrados, de sus posiciones, de sus intereses y estrategias (2005, p. 731).

La legislación, las normativas, las prescripciones, las demandas de la comunidad educativa, los objetivos, los resultados esperados, las metas propuestas. Un sinnúmero de propósitos a cumplir y lineamientos a seguir provenientes tanto de la administración como del contexto inmediato, modelan la tarea cotidiana de los directivos y se presentan como fuentes de regulación que se superponen unas a otras. 


\subsection{Multirregulación en la dirección escolar}

Siguiendo esta línea de pensamiento, João Barroso (2004) sostiene que la dirección escolar se encuentra entonces sujeta a una «mezcla híbrida en la regulación» (p. 124), y por lo tanto supeditada a diversas racionalidades. Licínio Lima (2011) se refiere a esta mixtura de racionalidades al afirmar que nos encontramos en una situación marcada por el hibridez en políticas educativas. Se producen medidas simultáneas y contradictorias: por un lado, el Estado es considerado el actor central en la provisión de los servicios y el encargado de brindar el financiamiento requerido. Asimismo, es el ente principal encargado de gestionar el control y la evaluación del accionar de las instituciones educativas. No obstante y paralelamente, se alienta la autonomía de las instituciones valorando su capacidad para autogestionarse. Mientras se producen procesos de centralización en la toma de decisiones y en la formulación de políticas, se invoca simultáneamente a la descentralización, a la autonomía de las escuelas, fuerte énfasis en la función del director como líder, desconfianza en los espacios colegiados y responsabilización de la familia.

Esta situación se refleja en procesos de multirregulación de la dirección escolar, al entender que el funcionamiento de la misma se establece por la interacción de diversos dispositivos de regulación y no solamente por la aplicación lineal de normas (Barroso, 2005). De esta manera, en la dirección escolar se entrecruza una regulación de tipo burocrática administrativa - el director como representante de la administración-, una regulación profesional - el director como representante del cuerpo docente- y una nueva regulación post-burocrática, basada en el conocimiento para el control por los resultados educativos, la calidad de los procesos y el éxito de la organización escolar en su conjunto.

Progresivamente y a la par de las reformas administrativas del Estado,

el control a priori, por las normas, es sustituido por el control a posteriori, por los resultados. El control del cumplimiento de la norma se mixtura lentamente con un control sobre lo producido, al considerar que son los resultados los que posibilitan dar cuenta del cumplimiento de lo reglado, posibilitan monitorear todo el proceso de forma eficaz y concreta sin necesidad de realizar un seguimiento de cada tarea o proceso. Se asiste igualmente al desenvolvimiento de una «regulación por los instrumentos» (Barroso, 2012, pág.5),

que se convierten en estrategias para regular en dos vías: encauzan las acciones hacia los resultados esperados y provoca en los sujetos la reorganización de sus acciones en función de esos instrumentos reguladores.

Barroso (2012) reconoce como instrumentos de regulación a las buenas prácticas, a los contratos, a la evaluación, a los proyectos, entre muchos otros. Una gestión democrática- profesional, o de tipo colegiada participativa se superpone con una gestión de tipo postburocrática (Barroso, 2011) tecnocrática y racionalista que puede transformarse en una gestión post-democracia gerencial (Lima, 2011). En esta regulación post-burocrática, el directivo se vincula con la figura del gerente de empresa, se lo responsabiliza por la mejora institucional, se espera de él una vocación política, perfil administrativo, un líder empático y carismático, gestor del cambio. Se deposita en una figura unipersonal, procesos que deberían ser colectivos. Se produce entonces lo que Licínio Lima (2011) caracteriza como hiperburocracia, es decir, un aumento exponencial de la burocracia escolar que implica

la sustitución del liderazgo colegial por el liderazgo unipersonal [...] la centralización y concentración de poderes de decisión; el regreso a la organización en línea, la mayor jerarquización y la división del trabajo entre directores y profesores; la creciente relevancia del saber experto y del poder de la tecnoestructura, de los suplentes y asesores, de las instancias especializadas en la prestación de servicios técnicos; la obsesión por la eficacia y eficiencia, por la elección óptima y por la performance competitiva; la centralidad de los procesos de gestión de la calidad, de evaluación y de medición, bajo inspiración neopositivista (rankings, escuelas de excelencia, evaluación externa, test estandarizados, padrones, etc.) (p. 11). 


\section{PLANES, PROGRAMAS Y PROYECTOS EN LA ESCUELA: ¿INCLUSIÓN EDUCATIVA O NUEVAS ESTRATEGIAS DE CONTROL?}

Los planes de acción a largo y medio plazo, los programas focalizados que pretenden cubrir necesidades, atender problemáticas específicas y fortalecer iniciativas particulares y los proyectos temáticos que pretenden trabajar con un contenido o temática situado en el corto plazo, se mixturan con los requerimientos a cumplir por las escuelas según lo establecido por las normativas que dimanan de los órganos de gobierno.

Una de las características de estos planes y programas es su pertenencia a diferentes jurisdicciones y niveles de gobierno, con su consecuente diversidad de lógicas de funcionamiento, evaluación, rendición de cuentas y monitoreo.

Se introducen en las escuelas nuevas tareas, nuevos formatos de trabajo, nuevas exigencias y objetivos a cumplir y por tanto, nuevas formas de control. En numerosas ocasiones, estas acciones focalizadas implican la incorporación de nuevos roles al cotidiano escolar: tutores; coordinadores de ciclos, de tutores, de cursos; coordinadores de programas y proyectos: técnicos territoriales, orientadores, mediadores, talleristas e incluso practicantes de múltiples instituciones y disciplinas. Estas figuras se suman a los jefes de estudio, psicopedagogos, psicólogos educativos, preceptores, celadores y secretarios, figuras que se pueden considerar tradicionales en el formato escolar. La tarea de coordinación, supervisión de las tareas, así como la exigencia de respuestas a cada demanda particular, recaen en la dirección escolar, reorganizando el cotidiano de las instituciones educativas.

La vicedirectora de la escuela de la ciudad de Córdoba expresa esta realidad de la siguiente manera: "Yo tengo muchos jefes: jefe de Programa de Mejoras, de Plan FinES, CAJ, Conectar Igualdad, PIT, y Supervisión que queda en último plano, por todos estos planes y programitas ${ }^{1} »$ (Entrevista 1, Vicedirectora, Escuela 1).

La gestión por planes y proyectos pese a que se presenta como propuesta superadora de la rigidez de la planificación clásica, encierra una nueva forma de controlar y orientar la labor de las escuelas hacia el cumplimiento de objetivos planteados por fuera de los centros educativos. En el caso de la escuela de la ciudad de Málaga, el instituto educativo lleva adelante un gran número de programas y proyectos, algunos ofrecidos por la Junta de Andalucía y otros por iniciativa particular de la escuela ${ }^{2}$. Según las observaciones realizadas en el trabajo de campo, fue posible constatar el funcionamiento efectivo de un sinnúmero de proyectos, la sucesión de actividades y la obligatoria presentación de resultados de cada iniciativa. El Equipo Técnico de Coordinación Pedagógica (ETCP, órgano máximo de coordinación) en una de sus reuniones periódicas, da cuenta de la consolidación de los programas y proyectos como nuevo instrumento de regulación: "la idea es mantener lo que hay y agregar más ofertas» (Profesor, en reunión de ECTP).

Son los directivos escolares quienes cargan sobre sus espaldas la responsabilidad de poner en marcha las acciones específicas del plan o programa, capacitar a los miembros de la comunidad educativa para la ejecución de las actividades e interpretar las lógicas de cada programa para adecuarlo a la realidad específica de la escuela. Asimismo deben realizar la evaluación del cumplimiento de los objetivos propuestos, presentar los resultados obtenidos con la implementación, velar por la seguridad de los materiales que pueda haber recibido la institución como parte del programa, rendir cuentas por la inversión del dinero que ingresó a la institución y/o generar estrategias alternativas para la búsqueda de recursos y evitar que los programas sean retirados de las instituciones. «Si el objetivo es que los chicos estén ocupados todo el tiempo, que nos paguen por cada programa, porque todo es por el mismo precio» (Charla informal, Vicedirectora, Escuela 1).

\footnotetext{
${ }^{1}$ Los programas y proyectos en funcionamiento en el IPEM de la ciudad de Córdoba en los años académicos 2014-2015 fueron: Plan de Mejora Institucional, Centro de Actividades Juveniles (CAJ), Conectar Igualdad, Plan Plan de Finalización de Estudios Primarios y Secundarios para Jóvenes y Adultos (FinES), Programa de Inclusión/Terminalidad de la Escuela Secundaria 14-17 (PIT).

2 Los programas y proyectos en vigencia en la escuela de la ciudad de Málaga en el año académico 2015-2016 fueron: Huerto Escolar, Sobre Ruedas, Recapacicla, Escuela TIC 2.0, Escuela Espacio de Paz, Plan de Igualdad, Acompañamiento Escolar, Educación Financiera, Plan de Autoprotección, Forma Joven, Escuelas Deportivas, Apoyo Lingüístico a Inmigrantes, Plan de Compensación Educativa, Proyecto Profundiza, Escuela de Madres y Padres, Programa de Mediación, Proyecto Comenius y Asociación Senderista Realenga.
} 
De esta manera, se generan nuevos modos de control, direccionamiento y seguimiento de las acciones de los centros escolares a través de nuevos instrumentos y mecanismos focalizados.

\section{DISCUSIÓN Y CONCLUSIONES}

Son numerosas las estrategias que regulan la tarea de los directivos escolares en arreglo a una gestión de tipo postburocrática, es decir, centrada en los resultados (Barroso, 2011). Mejorar el rendimiento de los alumnos, alcanzar resultados que mejoren las estadísticas, destacar en la ejecución de proyectos focalizados y obtener excelentes puntajes en pruebas estándares, son sólo algunos de los ejemplos de la lógica de producción mercantil en los sistemas educativos. Estos mecanismos alientan la competencia entre las instituciones educativas, generan individualismo, profundiza las diferencias entre los circuitos de formación, reorienta el proceso de aprendizaje en función de la evaluación y perjudica seriamente a las escuelas que atienden a poblaciones más desfavorecidas. La toma de decisiones en los centros educativos fluctúa en arreglo a los posibles beneficios, en especial económicos, que se puedan obtener (Torres Santomé, 2010).

Liderazgo, entrenamiento de competencias, trabajo con proyectos, adopción de buenas prácticas,resolución de conflictos, incentivos al desempeño, innovación y procesos de mejora continua, se transforman en los mecanismos liderados por los directores escolares para asegurar la eficacia educativa. Garantizar la productividad en el centro escolar, desplaza progresivamente del foco a la educación como objetivo central de la escuela.

El monitoreo y control de establecen de forma permanente mediante el accountability y la rendición de cuentas, la evaluación adopta numerosas formas y se extiende a todos los miembros de la comunidad educativa, bajo el justificativo de la búsqueda de calidad y excelencia, que se miden mediante índices de calidad y posicionan a los centros educativos enla carrera por optimizar su tarea para competir en el mercado educativo. El alcance de los planes, programas y proyectos, y los resultados obtenidos con la implementación de cada uno de ellos, son fácilmente traducibles en indicadores, en parámetros objetivables y en índices medibles. Por tanto, una gestión escolar centrada en proyectos, es una gestión susceptible de ser evaluada cuantitativamente, en función del rendimiento y la productividad, alentando procesos de calificación, catalogación, elaboración de rankings y circuitos diferenciados. Se generan escuelas más y menos eficientes, más y menos atractivas, más y menos productivas. Esto sin dudas, aleja progresivamente a la dirección escolar de sus objetivos centrales: brindar las condiciones necesarias para que de todos los sujetos sin distinción puedan acceder a la escolaridad obligatoria y reciban una educación de calidad que les posibilite un desarrollo integral.

\section{REFERENCIAS BIBLIOGRÁFICAS}

Barroso, J. (2012). A direcção da escola - tensões no presente, desafios para ofuturo. Ponencia presentada al III Congresso Ibero-Americano de Política e Administração da Educação. Zaragoza, España.

Barroso, J. (2011). Direcção de escolas e regulação das políticas: em busca do unicórnio. En A. Neto-Mendes, J. A. Costa y A. Ventura (Eds.). A emergencia do diretor da escola: questões políticas e organizacionais, (11-22). Aveiro: Universidad de Aveiro.

Barroso, J. (2005). O Estado, a educação e a regulação das políticas públicas. Revista Educação \& Sociedade, Campinas, 26(92),725-751.

Barroso, J. (2004). La autonomía de las escuelas en el contexto de cambio de los modos de regulación de las políticas y de la acción educativa: el caso portugués. Revista de Educación, 33, 117-140.

Lima, L. (2011). Políticas educacionais, organização escolar e trabalho dos profesores. Teoria e Pratica, 38(21),1-18. 
Miranda, E. y Lamfri, N. (2008). Los efectos de la multirregulación política en educación en la configuración de nuevas condiciones de trabajo e identidad del oficio docente. Cuadernos de Educación, 6(6),197-210.

Torres Santomé, J. (2010). La justicia curricular. El caballo de Troya de la cultura escolar. Madrid: Ediciones Morata. 